\title{
Sleep Contributes to the Strengthening of Some Memories Over Others, Depending on Hippocampal Activity at Learning
}

\author{
Géraldine Rauchs, ${ }^{1,2}$ Dorothée Feyers, ${ }^{1,3}$ Brigitte Landeau, ${ }^{2}$ Christine Bastin, ${ }^{1}$ André Luxen, ${ }^{1}$ Pierre Maquet, ${ }^{1}$ \\ and Fabienne Collette ${ }^{1,3}$ \\ ${ }^{1}$ Cyclotron Research Centre, University of Liège, 4000 Liège, Belgium, ${ }^{2}$ Inserm-Ecole Pratique des Hautes Etudes-University of Caen Basse-Normandie, \\ Research Unit U923, Groupement d'Intérêt Public Cyceron, 14074 Caen, France, and ${ }^{3}$ Cognitive and Behavioural Neuroscience Centre, University of Liège, \\ 4000 Liège, Belgium
}

Memory consolidation benefits from sleep. In addition to strengthening some memory traces, another crucial, albeit overlooked, function of memory is to erase irrelevant information. Directed forgetting is an experimental approach consisting in presenting "to be remembered" and "to be forgotten" information that allows selectively decreasing or increasing the strength of individual memory traces according to the instruction provided at learning. This paradigm was used in combination with functional MRI to determine, in humans, what specifically triggers at encoding sleep-dependent compared with time-dependent consolidation. Our data indicate that relevant items that subjects strived to memorize are consolidated during sleep to a greater extent than items that participants did not intend to learn. This process appears to depend on a differential activation of the hippocampus at encoding, which acts as a signal for the offline reprocessing of relevant memories during postlearning sleep episodes.

\section{Introduction}

Ample evidence indicates that sleep favors the consolidation of newly acquired information in memory (Born et al., 2006). Unlike memory consolidation, forgetting is often considered as a nuisance or a default of memory functioning. Yet, forgetting can also be a positive and intentional act, crucial for a proper functioning of memory enabling to update or to erase irrelevant information. The psychologist and philosopher William James wrote that "in the practical use of our intellect, forgetting is as important a function as recollecting" (James, 1892, p. 679). Nearly a century later, Crick and Mitchinson (1983) proposed that sleep might contribute to the forgetting of the weakest or irrelevant memory traces.

Although the mechanism by which recently acquired memory traces are consolidated during sleep is increasingly better understood (Diekelmann and Born, 2010), some issues remain unsolved. Thus, combined with functional MRI (fMRI), we used a directed forgetting paradigm, known to selectively decrease or reinforce individual memory traces, to causally determine how the quality of the initial memory trace at encoding influences the off-line reprocessing of recently acquired memories during sleep. During an encoding fMRI

Received July 30, 2010; revised Dec. 13, 2010; accepted Dec. 15, 2010.

This research was supported by the Belgian Fonds National de la Recherche Scientifique, the Fondation Médicale Reine Elisabeth, the Research Fund of the University of Liège, and Interuniversity Attraction Poles ProgrammeBelgian State-Belgian Science Policy.

Correspondence should be addressed to Dr. Fabienne Collette, Senior Research Associate, Neuropsychology Unit, Boulevard du rectorat 3 (B33), 4000 Liège, Belgium. E-mail: f.collette@ulg.ac.be.

DOI:10.1523/JNEUROSCI.3972-10.2011

Copyright $\odot 2011$ the authors $\quad 0270-6474 / 11 / 312563-06 \$ 15.00 / 0$ session, young healthy participants learned a series of words. Each word was followed by an instruction indicating whether the item was "to be remembered" (TBR item) or "to be forgotten" (TBF item). It was stressed that the subsequent memory test would only be based on TBR words. Participants were subsequently pseudorandomly assigned to one of two groups in which they were either allowed to have regular sleep (RS group) or were totally sleep deprived (TSD group) during the postlearning night. Three days after encoding, during another fMRI session, memory for TBR and TBF items was probed using a recognition task during which subjects had to categorize each word presented as previously encountered (whatever the instruction at encoding) or new. We hypothesized that RS participants would recognize more TBR than TBF items. Given that previous studies using recognition tasks reported only moderate or no beneficial effect of sleep on memory performance (Diekelmann et al., 2009), the recognition rate for TBR items was expected to be equal or marginally smaller in TSD than in RS participants. In contrast, sleep deprivation should increase memory errors (Diekelmann et al., 2008) and favor the recognition of TBF items. As sleep-dependent memory consolidation is assumed to imply a replay of hippocampal activity (Buzsáki, 1996), leading to the progressive transfer of the memory burden from hippocampo-neocortical to predominantly neocortical long-term stores (Squire and ZolaMorgan, 1991; Frankland and Bontempi, 2005), we expected larger hippocampal responses during retrieval of TBR compared with TBF items in RS participants, and also for TBR items that were later consolidated during sleep compared with forgotten ones. 


\section{Materials and Methods}

Subjects. Twenty-six right-handed volunteers (11 males, 15 females, mean age: $23.1 \pm 2.7$ years) gave their written informed consent to participate in this study, which was approved by the Ethics Committee of the Faculty of Medicine of the University of Liège. None of the subjects reported any history of trauma or medical, psychiatric, or sleep disorders, nor disturbances of their sleep-wake cycle during the last 6 weeks. Structural MRI was normal on visual inspection. Self-report questionnaires assessed sleep quality (Buysse et al., 1989) and circadian typology (Horne and Östberg, 1976).

Volunteers followed a constant sleep schedule (according to their own sleep-wake schedule $\pm 1 \mathrm{~h}$ ) $3 \mathrm{~d}$ before the first visit and kept this schedule for 3 more days, until their second visit.

After encoding, participants were pseudorandomly assigned to one of the two following groups (Fig. 1). In the sleep group ( $n=14$, six males, eight females; RS group), subjects were allowed to sleep at home following their regular habits for the three postlearning nights. In the sleep-deprived group ( $n=12$, five males, seven females; TSD group), subjects remained awake in the laboratory during the first postlearning night. During this night, participants' physical activity was maintained as low as possible. Subjects remained most of the time in a sitting position and played quiet games or watched movies under constant supervision by the experimenters. Food intake was standardized across subjects, and luminance exposure was kept $<8$ lux. At 8:00 A.M., subjects were allowed to leave the laboratory. They were instructed to follow their usual daytime activities and to abstain from napping during the day. All subjects slept as usual at home on the second and third postlearning nights. Sleep quality for each night from before the learning session to before the testing session was assessed using a standardized questionnaire (Ellis et al., 1981).

Stimuli and procedure. Stimuli were six-letter words selected from the Brulex French lexical database (Content et al., 1990). During the learning phase, subjects saw a series of one hundred words, one at a time. Fifty words were categorized as TBR items and the other as TBF items. TBR and TBF words were counterbalanced across subjects. Lexical frequency was controlled so that there were no difference between TBF and TBR items (TBR, 697.5 \pm 849.7; TBF, $697.7 \pm 681.1 ; F=0.0001, p>0.99$ ). Fifty control items (series of six crosses) were also presented in the encoding phase. The three types of items (TBR, TBF, and crosses) were pseudorandomly organized so that there were no more than three items of the same type consecutively.

Figure 1 illustrates the experimental procedure. The learning phase took place on day 1 and began by the presentation of a white fixation cross on the center of the screen for $800 \mathrm{~ms}$ and was followed by a black screen for $200 \mathrm{~ms}$. Then subjects saw a target item for $1 \mathrm{~s}$ and were instructed to mentally read the word presented. Next, an instruction "To remember" or "To forget" was displayed in yellow for $3 \mathrm{~s}$. If a remember instruction was given, participants were instructed to encode the item. If a forget instruction was presented, subjects were asked to engage suppression processes not to encode this particular word. Control items were presented according to the same procedure and the instruction was replaced by a succession of crosses. To be sure that subjects understood the instructions, an example with three items was shown before encoding, outside the scanner.

The recognition task was conducted on day 4 after two recovery nights. The 100 target stimuli were mixed with 100 distracters of equal lexical frequency (target items, $697.6 \pm 766.1$; distracter items, $698.6 \pm 606.3 ; F<0.001, p>0.99)$. Subjects first saw a white fixation cross displayed in the center of the screen for $500 \mathrm{~ms}$, followed by the presentation of a target or a distracter item for $5 \mathrm{~s}$. Subjects had to indicate, by pressing keys and in no more than $5 \mathrm{~s}$, if they had already seen the word or not, regardless the instruction given at learning. When control items were presented, subjects had to press one button or the other.

After retrieval, subjects filled out a debriefing questionnaire in which they had to explain the strategies used to memorize TBR items and forget TBF words. More precisely, they had to estimate on a five-point scale their use of various strategies (such as mental imagery, rehearsal of one or several words, and association with personal events or memories). A score of 1 indicated that they never used the strategy and 5 that they always used it. They could also indicate the use of a different strategy than those proposed by experimenters. Repetition of words during the $3 \mathrm{~d}$ interval between encoding and retrieval was also quantified using a four-point scale (from never to $>10$ times). No subject intensively rehearsed encoded items during the retention interval. Consequently, no item in any subject was removed from the analyses (supplemental Results, available at www.jneurosci. org as supplemental material).

fMRI data acquisition and analyses. Whole-brain functional $\mathrm{T} 2{ }^{*}$ weighted MRI data were acquired using a $3 \mathrm{~T}$ scanner (Siemens Allegra) using a gradient-echo planar imaging (EPI) sequence [32 transverse slices with $30 \%$ gap; voxel size, $3.4 \times 3.4 \times 3 \mathrm{~mm}^{3}$; repetition time (TR), $2130 \mathrm{~ms}$, echo time (TE), $40 \mathrm{~ms}$; flip angle, $90^{\circ}$; field of view $\left(\right.$ FoV), $220 \times 220 \mathrm{~mm}^{2}$ ]. For anatomical reference, a structural MR scan was acquired for each subject (T1-weighted 3D MPRAGE sequence; TR, $1960 \mathrm{~ms}$; TE, $4.43 \mathrm{~ms}$; inversion time, $1100 \mathrm{~ms}$; FoV, $230 \times 173 \mathrm{~mm}^{2}$; matrix size, $256 \times 192 \times 176$; voxel size, $0.9 \times$ $0.9 \times 0.9 \mathrm{~mm}^{3}$ ). Head movements were minimized by restraining the subject's head using a vacuum cushion. Stimuli were displayed on a screen positioned at the rear of the scanner, which the subject could comfortably see through a mirror mounted on the standard head coil.

Functional volumes were preprocessed and analyzed using SPM5 (www.fil.ion.ucl.ac.uk). The three initial volumes of each session were discarded to avoid T1 saturation effects. Volumes were realigned 
using iterative rigid body transformations that minimized the residual sum of square between the first and subsequent images. Volumes were spatially normalized to the MNI EPI template and spatially smoothed with a $8 \mathrm{~mm}$ full-width at half maximum (FWHM) Gaussian kernel.

Data were processed using two-step analysis, taking into account the intraindividual and interindividual variances. For each subject, changes in brain regional responses were estimated at each voxel using a general linear model.

During the encoding session, four trial types were modeled, as follows: TBR items recognized at the retrieval session (TBR hits), TBR items not recognized during retrieval (TBR misses), TBF items subsequently retrieved (TBF hits), and TBF items not retrieved (TBF misses). During the retrieval session, six trial types were modeled, as follows: TBR hits, TBR misses, TBF hits, TBF misses, correct rejections (distracter items considered as new), and false alarms (new items categorized as previously encountered). For each trial type, a given item was modeled as a delta function representing its onset. The ensuing vector was convolved with the canonical hemodynamic response function and was used as a regressor in the individual design matrix. Movement parameters estimated during realignment (translations in $x, y$, and $z$ directions and rotations around $x, y$, and $z$ axes) and constant vector were also included in the matrix as a variable of no interest. High-pass filter was implemented using a cutoff period of $128 \mathrm{~s}$ to remove the low-frequency drifts from the time series. Serial autocorrelations were estimated with a restricted maximum likelihood algorithm using an autoregressive model of order 1 (plus white noise).

For the encoding session, linear contrasts estimated the effect of instruction on the processing of items at encoding (TBR > TBF; TBF $>$ TBR) and the effect of successful encoding or unsuccessful active forgetting at encoding (TBR hits $>$ TBR misses; TBF hits $>$ TBR misses). For the retrieval session, the linear contrasts performed estimated the effect of successful retrieval (TBR hits $>$ TBR misses) and of unsuccessful forgetting (TBF hits $>$ TBF misses). The individual summary statistical images were spatially smoothed with a $6 \mathrm{~mm}$ FWHM Gaussian kernel and used in a second-level, random-effect analysis to account for intersubject variance in each contrast of interest. This analysis consisted of one-sample $t$ tests testing for the effect of interest in each group and two-sample $t$ tests comparing the responses between the two groups. Inclusive and exclusive masks were created with SPM maps thresholded at $p<0.001$ and $p<0.05$, respectively. Corrections for multiple testing were applied where mentioned by using either the family-wise error (FWE) correction over the whole brain or small volume correction (SVC) over small spherical volumes of interest (radius $10 \mathrm{~mm}$ ) around a priori locations of structures of interest taken from the literature.

\section{Results}

\section{Behavioral results}

Behavioral data of one subject in the TSD group was not included in the analyses due to an abnormal proportion of false alarms. Nevertheless, adding or removing data of this subject did not change the results for TBR hits and TBF hits. Proportions of "old" responses to TBR, TBF, and new items were compared using $t$ tests. This analysis revealed that TSD participants recognized as many TBR items as did RS participants [mean values $( \pm$ SEM): RS group, $0.71 \pm 0.03$; TSD group, $\left.0.76 \pm 0.03 ; t_{(23)}=1.32, p>0.099\right]$, but recognized more TBF items (RS group, $0.42 \pm 0.03$; TSD group, $0.58 \pm 0.03 ; t_{(23)}=$ $-3.38, p<0.001$ ) and made more false alarms (RS group, $0.25 \pm 0.04$; TSD group, $\left.0.44 \pm 0.06 ; t_{(23)}=2.68, p<0.01\right)$. These results could not be accounted for by persisting effects of sleep deprivation during recognition. Indeed, median reaction times in a psychomotor vigilance task [adapted from
Dinges and Powell (1985)], in which simple reaction times spaced by variable intervals (2-9s) are measured over a period of $10 \mathrm{~min}$, did not differ between sessions (encoding/recognition) and groups [encoding (mean \pm SD): RS, $262.9 \pm 21.8$ $\mathrm{ms}$; TSD, $271.5 \pm 24.1 \mathrm{~ms}$; recognition: RS, $264.8 \pm 18.1 \mathrm{~ms}$; TSD, $276.8 \pm 28.4 \mathrm{~ms}$; all $p$ values $>0.14]$.

However, as the global increase of "old" responses in the TSD group could indicate the existence of a response bias, data were further analyzed using the discrimination score $\left(\mathrm{d}^{\prime}\right)$ and the response criterion $(\mathrm{C})$, measures derived from the signal detection theory (Macmillan and Creelman, 1991). As d' and $\mathrm{C}$ for TBR and TBF items are not totally independent measures (both taking into account false alarms), statistical analyses were conducted using separate $t$ tests for TBR and TBF items. Recognition accuracy was equivalent between groups for TBR [RS group (mean \pm SEM), $1.33 \pm 0.7$; TSD group, $1.1 \pm 0.68$ $\left.t_{(23)}=-0.79, p>0.22\right)$ and TBF items (RS group, $0.52 \pm 0.33$; TSD group, $\left.\left.0.49 \pm 0.26 ; t_{(23)}=-0.21, p>0.42\right)\right]$. However, similar analyses on response criteria for TBR and TBF items yielded significant group differences (mean $\mathrm{C} \pm$ SEM: TBR items: RS group, $0.1 \pm 0.2$; TSD group, $-0.21 \pm 0.34 ; t_{(23)}=$ $-2.76, p<0.01$; TBF items: RS group, $0.48 \pm 0.33$; TSD group, $\left.0.06 \pm 0.3 ; t_{(23)}=-3.31, p<0.01\right)$, indicating that TSD participants had a more lenient response criterion than participants in the RS group.

Similar analyses conducted on mean response times failed to reveal any effect of group $(p>0.3)$. Distinguishing between the different types of items and possible responses (TBR hits, TBR misses, TBF hits, TBF misses, and hits items categorized as previously encountered or not), statistical analyses revealed similar results (all $p$ values $>0.25$ ). Thus, our data indicate that memory performance differed between TSD and RS participants (for TBF items and false alarms) without any significant difference either in recognition accuracy or in response times. Together with the results of the psychomotor vigilance task, these data exclude the possibility that this difference in memory performance was merely due to an effect of tiredness in TSD participants.

The use of various strategies was estimated by means of a five-point scale. To memorize TBR items, participants used mainly a rehearsal strategy of one (mean score \pm SD, $4.19 \pm 1.06$ ) or several $(3.61 \pm 1.10)$ items, created association between items to form a short story or a sentence $(3.61 \pm 1.3)$, and /or tried to associate the words to memories or personal events $(3.58 \pm 1.42)$. Mental imagery was less often used by the participants $(2.38 \pm$ 1.1). When a forgetting instruction was displayed, participants mainly rehearsed the TBR items presented before $(3.58 \pm 1.36)$ and/or tried to think to nothing in particular $(2.92 \pm 1.41)$. As with TBR items, mental imagery of TBF items was rarely used by participants $(2.19 \pm 1.36)$.

Finally, statistical analyses were conducted to compare mental repetitions between groups (using Mann-Whitney $U$ tests) and to compare each type of item (TBR/TBF) within each group (using Wilcoxon tests). These analyses indicate that mental repetitions did not differ between TSD and RS participants for TBR and TBF items (all $p$ values $>0.1$ ). As expected, and consistent with the analysis of the strategies used to memorize or forget words, TBR items were more repeated during the retention interval. More precisely, within the RS group, there was a significant difference between TBR and TBF items regarding the first point of the scale ("item 


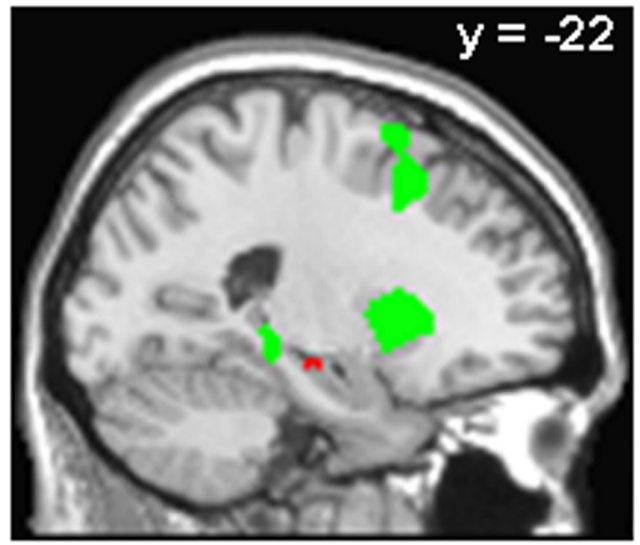

Figure 2. Higher responses at encoding for TBR compared with TBF items (green) and for TBR hits compared with TBR misses (red) in RS but not in TSD participants (exclusive masking at $p<0.05$ ). Responses are displayed on a sagittal section of the MNI template at $p<0.001$, uncorrected.

never repeated during the $3 \mathrm{~d}$ interval"; TBF, $80.4 \%$; TBR, $59.7 \% ; p<0.001$ ) and for the second point ("repeated between 1 and 5 times"; TBF, 17.4\%; TBR, 29.1\%; $p<0.01$ ). As for the TSD group, there was also a significant difference between TBR and TBF items for the first point of the scale (TBF, $84 \%$; TBR, $68.8 \% ; p<0.05$ ) and for the third point ("repeated between 5 and 10 times"; TBF, 1.2\%; TBR, 7.7\%; $p<0.05)$. It is worth noting that there is no between-group difference concerning the fourth point of the scale ("repeated $>10$ times"), which could have be a potential confound in fMRI analyses.

\section{fMRI results}

Compared with TBF items and regardless of their status at retrieval (recognized or forgotten), encoding of TBR items activated, in both groups, a set of brain regions including frontal areas, the thalamus and putamen, and the left posterior hippocampus (all $p$ values $<0.05$, corrected for multiple comparisons) (Fig 2, supplemental Table 1, available at www. jneurosci.org as supplemental material). The reverse contrast $(\mathrm{TBF}>\mathrm{TBR})$ revealed mainly frontal and posterior cortical activations but none of them survived correction for multiple comparisons.

Next, we compared brain activity associated, in both groups, to the encoding of TBR items that were later consolidated (TBR hits) compared with TBR items that were forgotten (TBR misses). In the RS group, this contrast revealed only higher activity in the left hippocampus $\left(p^{\mathrm{svc}(10 \mathrm{~mm})}<0.05\right)$. Interestingly, parameter estimates indicated that hippocampal response in RS participants was larger for TBR hits (mean \pm SEM, $0.76 \pm 0.32$, arbitrary units) compared with TBR misses $(0.35 \pm 0.29)$, and also for TBF hits $(0.67 \pm 0.33)$ compared with TBF misses $(0.44 \pm 0.21)$. The same analysis in the TSD group failed to reveal any significant response. To determine whether hippocampal activation at encoding specifically triggers sleep-dependent consolidation and not time-dependent consolidation, we masked the contrast in the RS group by that in the TSD group (exclusive masking, $p<0.05$ ), revealing that the hippocampal response was indeed significant in RS but not in TSD participants ( $p^{\mathrm{svc}(10 \mathrm{~mm})}$ $<0.05$ ) (Fig. 2). The reverse contrast (contrast in TSD subjects with exclusive masking by the contrast in RS participants) failed to reveal any significant response.
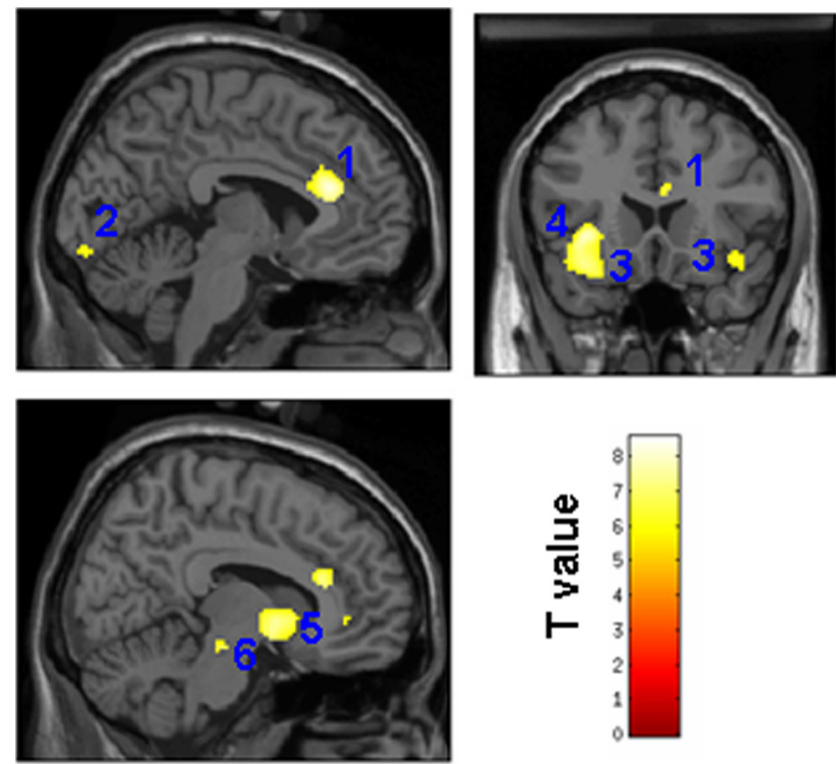

Figure 3. Common brain activations at retrieval for TBR hits and TBF hits in the RS group (compared with correct rejections). Activations are displayed at $p<0.05$ (FWE corrected) on sections of the MNI template. 1, Anterior cingulate cortex; 2, calcarine region; 3, insula; 4, inferior frontal gyrus; 5 , caudate nucleus; 6 , lenticular nucleus.

Similar analyses were conducted to compare TBF hits to TBF misses. In RS group (but not in TSD; exclusive masking, $p<$ $0.05)$, this analysis did not reveal any significant response. In contrast, thalamic responses were bilaterally significant ( $[10-30$ 4], $[-6-306], p$ FWE corrected $<0.05)$ in TSD but not in RS participants (exclusive masking, $p<0.05$ ).

fMRI data acquired during the recognition session were analyzed to assess a different processing of TBR and TBF items during sleep and nocturnal wakefulness. We compared the brain activity associated with correct item recognition (hits) to that of forgotten items (misses) separately for TBR and TBF items. In the RS group, but not in the TSD one (exclusive masking, $p<0.05$ ), TBR hits elicited more activity than TBR misses in a large neural network including frontal and posterior cortical areas (precuneus, lingual gyrus, superior parietal lobule) as well as in the amygdala, putamen, and cerebellum (supplemental Table 2, available at www.jneurosci.org as supplemental material). In the TSD group (but not in the RS one; exclusive masking, $p<0.05$ ), no activation survived correction for multiple comparisons. Similar analyses for TBF items (TBF hits $>$ TBF misses) revealed significant differential responses in RS but not in TSD subjects (exclusive masking, $p<0.05$ ) in the superior temporal gyrus and in frontal areas (medial frontal and anterior cingulate gyri), as well as in the thalamus (all $p$ values $<0.05$, corrected for multiple comparisons) (supplemental Table 3, available at www.jneurosci. org as supplemental material). The reverse contrast (TSD > RS; exclusive masking at $p<0.05$ ) failed to reveal any significant response.

Finally and importantly, we wondered whether sleep lead to a similar processing of TBR hits and TBF hits. To determine this, we masked, in each group separately, the contrast of TBR hits versus correct rejections by the contrast of TBF hits versus correct rejections (inclusive masking, $p<0.001$ ). In $\mathrm{RS}$ participants, this analysis revealed common responses to recognition for both types of items (compared with correct rejections) in the anterior cingulate cortex and insula, the parahippocampal gyrus, the inferior frontal gyrus, various posterior cortical areas (lingual 
gyrus, precuneus, calcarine region), the vermis, the putamen, and caudate nucleus (all $p$ values $<0.05$, corrected for multiple comparisons) (Fig. 3, supplemental Table 4, available at www. jneurosci.org as supplemental material). The same analysis with TSD participants failed to reveal any significant response surviving corrections for multiple comparisons.

\section{Discussion}

This study contributes to better understand the specific conditions in which sleep-dependent memory consolidation occurs. From a behavioral standpoint, recognition accuracy for TBR items was equivalent between groups, as previously reported in studies using recognition tasks (Diekelmann et al., 2009). Interestingly, TSD participants recognized significantly more TBF items than subjects in the RS group, indicating, at a behavioral level, that the status of engrams after encoding varies between TBR and TBF in such a way that if sleep subsequently occurs, the two types of memories are processed differently. However, it is worth noting that TSD participants also had a more lenient response criterion than subjects in the RS group, which could influence recognition performance.

To understand what triggers sleep-dependent memory consolidation, fMRI data obtained during encoding were analyzed. These data revealed higher hippocampal activity for TBR than for TBF items and, more interestingly, larger responses in a non-overlapping area for TBR hits compared with TBR misses (Fig. 2). More interestingly, these hippocampal responses to TBR items during encoding were observed in the RS group and not in the TSD group (exclusive masking). This finding suggests that hippocampal activation at learning specifically triggers sleepdependent compared with time-dependent memory consolidation. Thus, during sleep, items that subjects strived to memorize and were associated with stronger hippocampal activity are consolidated to a greater extend than items that participants did not attempt to learn. These results extend previous reports demonstrating that medial temporal activations at encoding predict subsequent remembering (Wagner et al., 1998; Eichenbaum et al., 2007). These results are also consistent with a recent study about false memories that indicates that lists of items that did not produce false memories differed from those that did by larger hippocampal responses at encoding (Darsaud et al., 2011). Collectively, these findings indicate that hippocampal activity during encoding crucially influences the offline processing of information during postlearning sleep and the later production of accurate, illusory, or unwanted memories.

Sleep-dependent memory consolidation is believed to rely on the coordinated replay of specific firing sequences between the hippocampus and the neocortex (Born et al., 2006). As already reported for motor sequence consolidation (Albouy et al., 2008) or false memories (Darsaud et al., 2011), our data suggest that large hippocampal responses at encoding might tag the neural populations in which sequence replay would preferentially occur during subsequent sleep, leading to their retrieval at retest regardless of whether they were initially to be remembered or forgotten.

The analysis of fMRI data obtained during the recognition task indicates that sleep and lack of sleep during the first postlearning night lead to a different processing of TBR and TBF items. Indeed, in the RS group but not in the TSD group (exclusive masking), correct recognition of TBR and TBF items was associated with significant responses in a set of neocortical regions including frontal areas (notably the anterior cingulate cortex) and temporal, parietal, and occipital areas, wherease TSD participants did not exhibit larger responses than in RS subjects (exclusive masking), whatever contrast was considered (supplemental Tables 2, 3, available at www.jneurosci.org as supplemental material). The activation of neocortical areas such as the anterior cingulate cortex has been previously observed during the accurate retrieval of declarative memories in the waking state in rodents (Maviel et al., 2004) and humans (Takashima et al., 2006). Finally, TBR and TBF items correctly recognized, which elicited high hippocampal activity at encoding, undergo a similar processing during postlearning sleep, as revealed by a common retrieval network for both types of items in RS participants. Interestingly, the same analysis performed in TSD participants did not revealed any common pattern of activation for TBR and TBF items (compared with correct rejections). These results suggest that sleep, contrary to the simple passage of time, promotes the binding of the elements constituting a memory (item and associated contextual information) across various neocortical areas. This large network could also reflect the establishment of multiple traces within neocortical areas, a key mechanism subserving memory consolidation (Frankland and Bontempi, 2005). By contrast, remaining awake all night might hinder such a reorganization of memory traces within the brain and possibly reflects an earlier stage of memory consolidation.

These results shed new light on the role of sleep in memory consolidation, providing evidence that during sleep, relevant items that subjects memorized are consolidated to a greater degree than irrelevant information that subjects do not intend to learn. This process, which hinders consolidation of irrelevant information in favor of pertinent items, is contingent upon a differential hippocampal activity at encoding between memories to strengthen and those to erase or weaken. Our results indicate that brain activity at learning is crucial to determine the fate of relevant and irrelevant memories during subsequent sleep episodes.

\section{References}

Albouy G, Sterpenich V, Balteau E, Vandewalle G, Desseilles M, Dang-Vu T, Darsaud A, Ruby P, Luppi PH, Degueldre C, Peigneux P, Luxen A, Maquet $P$ (2008) Both the hippocampus and striatum are involved in consolidation of motor sequence memory. Neuron 58:261-272.

Born J, Rasch B, Gais S (2006) Sleep to remember. Neuroscientist 12:410-424.

Buysse DJ, Reynolds CF 3rd, Monk TH, Berman SR, Kupfer DJ (1989) The Pittsburgh sleep quality index: a new instrument for psychiatric practice and research. Psychiatry Res 28:193-213.

Buzsáki G (1996) The hippocampo-neocortical dialogue. Cereb Cortex 6:81-92.

Content A, Mousty P, Radeau M (1990) Brulex: une base de données lexicales informatisées pour le français écrit et parlé. L'Année Psychologique 90:551-566.

Crick F, Mitchinson G (1983) The function of dream sleep. Nature 304:111-114.

Darsaud A, Dehon H, Lahl O, Sterpenich V, Boly M, Dang-Vu T, Desseilles M, Gais S, Matarazzo L, Peters F, Schabus M, Schmidt C, Tinguély G, Vandewalle G, Luxen A, Maquet P, Collette F (2011) Does sleep promote false memories? J Cogn Neurosci 23:26-40.

Diekelmann S, Born J (2010) The memory function of sleep. Nat Rev Neurosci 11:114-126.

Diekelmann S, Landolt HP, Lahl O, Born J, Wagner U (2008) Sleep loss produces false memories. PLoS One 3:e3512.

Diekelmann S, Wilhelm I, Born J (2009) The whats and whens of sleepdependent memory consolidation. Sleep Med Rev 13:309-321.

Dinges DF, Powell JW (1985) Microcomputer analyses of performance on a portable, simple visual RT task during sustained operations. Behav Res Methods Instrum Comput 17:652-655. 
Eichenbaum H, Yonelinas AP, Ranganath C (2007) The medial temporal lobe and recognition memory. Annu Rev Neurosci 30:123-152.

Ellis BW, Johns MW, Lancaster R, Raptopoulos P, Angelopoulos N, Priest RG (1981) The St. Mary's Hospital sleep questionnaire: a study of reliability. Sleep 4:93-97.

Frankland PW, Bontempi B (2005) The organization of recent and remote memories. Nat Rev Neurosci 6:119-130.

Horne JA, Östberg O (1976) A self-questionnaire to determine morningness/ eveningness in human circadian rhythms. Int J Chronobiol 4:97-110.

James W (1890) The principle of psychology, Vol. 1. New York: Holt.

Macmillan A, Creelman CD (1991) Detection theory: a user's guide. Cambridge: Cambridge UP.
Maviel T, Durkin TP, Menzaghi F, Bontempi B (2004) Sites of neocortical reorganization critical for remote spatial memory. Science 305:96-99.

Squire LR, Zola-Morgan S (1991) The medial temporal lobe memory system. Science 253:1380-1386.

Takashima A, Petersson KM, Rutters F, Tendolkar I, Jensen O, Zwarts MJ, McNaughton BL, Fernández G (2006) Declarative memory consolidation in humans: a prospective functional magnetic resonance imaging study. Proc Natl Acad Sci U S A 103:756-761.

Wagner AD, Schacter DL, Rotte M, Koutstaal W, Maril A, Dale AM, Rosen BR, Buckner RL (1998) Building memories: remembering and forgetting of verbal experiences as predicted by brain activity. Science 281 : $1188-1191$. 